\title{
Efeitos de citocininas sobre a anatomia foliar e o crescimento de Annona glabra L. durante o cultivo in vitro e ex vitro
}

\author{
Effects of cytokinins on the leaf anatomy and growth of Annona glabra L. during in vitro and \\ ex vitro culture \\ Lenaldo Muniz de Oliveira ${ }^{\text {I* }}$ Renato Paiva ${ }^{\text {II }}$ Magdi Ahmed Ibrahim Aloufa ${ }^{\text {III }}$ \\ Evaristo Mauro de CastroII José Raniere Ferreira de SantanaI Rairys Cravo NogueiraII
}

\begin{abstract}
O efeito de diferentes fontes de citocininas durante RESUMO o cultivo in vitro de A. glabra sobre características anatômicas de folhas e crescimento das plantas foi avaliado neste trabalho. BAP (6-benzilaminopurina) e KIN (cinetina) induziram aumento na espessura do mesofilo, enquanto que ZEA (zeatina) promoveu aumento na densidade e no índice estomático e no desenvolvimento do sistema vascular de folhas. A utilização de KIN e BAP proporcionou maior desenvolvimento e taxa de sobrevivência das plantas durante as fases de enraizamento $e$ aclimatização.
\end{abstract}

Palavras-chave: Annonaceae, morfogênese, aclimatização, cinetina, benzilaminopurina.

\section{ABSTRACT}

The effect of different sources of cytokinins during the in vitro cultivation of A. glabra on anatomical characteristics of leaves and plant growth was evaluated in this work. BAP (6benzilaminopurine) and KIN (kinetin) induced an increase in leaf mesophyll thickness, while the ZEA (zeatin) promoted an increase in density and stomatic index and development of leaves vascular system. The utilization of KIN and BAP improved higher plant development and survival rate during the acclimatization and rooting phases.

Key words: Annonaceae, morphogenesis, acclimatization, kinetin, benzilaminopurine.

Annona glabra L. é uma annonaceae de ocorrência natural na América do Sul, apresentando elevado potencial agronômico e fitoquímico. Essa espécie apresenta limitações na sua propagação sexuada, com germinação lenta, desuniforme e pouco expressiva. Nesse contexto, a micropropagação tem se mostrado como uma ferramenta viável para a propagação de espécies de anonáceas (LEMOS \& BLAKE, 1996; NAGORI \& PUROHIT, 2004). Contudo, a baixa taxa de brotação dos explantes e a elevada abscisão foliar têm limitado o uso dessa técnica nesse grupo de plantas.

Na micropropagação de anonáceas o BAP (6-benzilaminopurina) e KIN (cinetina) têm sido as fontes de citocininas mais utilizadas (NAGORI \& PUROHIT, 2004). ZEA (zeatina) foi utilizada na micropropagação juvenil de A. cherimola Mill., induzindo a formação de maior número de brotos por explante e folhas mais expandidas (ENCINA et al., 1994). TDZ (Thidiazuron), apesar de ser uma das mais ativas fontes de citocininas, especialmente para espécies lenhosas recalcitrantes, não tem sido usado na micropropagação de anonáceas. Neste trabalho serão apresentados os resultados obtidos com a utilização de quatro fontes de citocininas sobre características anatômicas das folhas de A. glabra e o desenvolvimento das plantas durante o cultivo in vitro e ex vitro.

Os explantes (segmentos nodais) foram desinfestados em água corrente por 6 horas e imersão

\footnotetext{
IUniversidade Estadual de Feira de Santana (UEFS), Departamento de Ciências Biológicas, Feira de Santana, BA, Brasil. Endereço para correspondência: Rua Oxossi Guerreiro, no 99, Bairro Sobradinho, 44020-270, Feira de Santana, BA, Brasil. E-mail: lenaldo@uefs.br.*Autor para correspondência.

"Universidade Federal de Lavras (UFLA), Lavras, MG, Brasil.

IIIUniversidade Federal do Rio Grande do Norte (UFRN), Natal, RN, Brasil.
} 
em álcool etílico $70 \%$ (v/v) por 1 minuto, hipoclorito de sódio $1 \%$ por 15 minutos, água destilada e autoclavada por três vezes de 1 minuto. Estes foram cultivados em meio WPM(LLOYD \& MCCOWN, 1980), suplementado com $30 \mathrm{~g} \mathrm{~L}^{-1}$ de sacarose, $1 \mathrm{~g} \mathrm{~L}^{-1}$ de carvão ativado, $500 \mathrm{mg} \mathrm{L}^{-1}$ de benlate e ágar (Sigma) a $0,65 \%$. Os tratamentos consistiram da adição de BAP, TDZ, KIN e ZEA, todos na concentração de $1,0 \mathrm{mg} \mathrm{L}^{-1}$, conforme resultados obtidos por SANTANA(2003). Após 55 dias de cultivo in vitro, foram coletadas folhas para análise anatômica.

Para análise anatômica, utilizou-se a metodologia descrita por JOHANSEN (1940). As seções transversais foram obtidas com o auxílio de um micrótomo manual com a inclusão do material em medula do pecíolo de imbaúba. Estes foram clarificados com hipoclorito de sódio $50 \%$, lavados em água destilada, corados com azul de astra e safranina e montados em glicerina 50\% (KRAUS \& ARDUIN, 1997). As lâminas com seções paradérmicas das faces abaxial e adaxial das folhas, obtidas à mão livre, foram montadas com solução corante de safranina $1 \% \mathrm{em}$ água glicerinada. As determinações da espessura dos tecidos foram realizadas com auxílio de uma ocular micrométrica acoplada em microscópio de luz. A contagem do número de estômatos foi realizada com o auxílio de uma câmara clara em microscópio OLYMPUS CBB e o cálculo do índice estomático (IE) foi realizado por meio da fórmula de CUTTER (1986). Foram avaliadas cinco folhas oriundas de cinco brotações diferentes, provenientes do quarto nó, avaliando-se quatro campos do terço mediano de cada folha.

As plantas micropropagadas foram enraizadas em meio WPM suplementado com $30 \mathrm{~g} \mathrm{~L}^{-1}$ de sacarose, $1 \mathrm{~g} \mathrm{~L}^{-1}$ de carvão ativado, $2 \mathrm{mg} \mathrm{L}^{-1}$ de AIB e pH ajustado para 5,0, segundo resultados obtidos por SANTANA (2003). A aclimatização foi realizada em substrato comercial Plantmax ${ }^{\circledR}$ durante 21 dias, sob fotoperíodo de 16 horas, irradiância de fótons de $55 \mu \mathrm{mol}$ $\mathrm{m}^{-2} \mathrm{~s}^{-1}$ e temperatura de $27 \pm 2^{\circ} \mathrm{C}$. No final das fases de enraizamento e de aclimatização, quantificaram-se a matéria seca, a área foliar e a taxa de sobrevivência das brotações, avaliando-se 25 plantas por tratamento.

Verificou-se neste trabalho que as plantas cultivadas na presença de TDZ, BAP e KIN apresentaram estômatos com maior diâmetro equatorial em relação às plantas submetidas aos tratamentos com
ZEA e sem adição de citocinina (Figura 1). ZEA proporcionou a maior densidade e o maior índice estomático. $\mathrm{O}$ aumento da relação entre o número de estômatos e o número de células epidérmicas, representado pelo aumento no índice estomático, nas folhas de plantas cultivadas em meio com ZEA, tem relação direta com a perda de água por parte das plantas durante a fase de aclimatização, sobretudo se o mecanismo de controle na abertura no e fechamento dos estômatos não seja adequadamente desenvolvido durante o cultivo in vitro. Sobre esse aspecto verificouse, nas plantas submetidas aos tratamentos com ZEA, a formação de estômatos com formatos mais arredondados e há evidências de uma menor funcionalidade dos estômatos que apresentam formas menos elipsóide (SCIUTTI \& MORINI, 1995), sendo uma possível explicação para a redução na taxa de sobrevivência durante a fase de aclimatização das plantas cultivadas na presença desses fitoreguladores.

As diversas fontes de citocininas utilizadas neste trabalho não promoveram efeitos significativos sobre a espessura das epidermes adaxial e abaxial das folhas de A. glabra (Figura 2). Por outro lado, a espessura dos parênquimas esponjoso e paliçádico e, conseqüentemente, do limbo foliar foram significativamente afetadas pela presença de BAP e KIN no meio de cultura (Figura 2). Nas folhas das plantas submetidas aos tratamentos com BAP e KIN, foi verificada, ainda, maior diferenciação do mesofilo, com reduzida proporção de espaços intercelulares, sugerindo uma maior eficácia dessas duas fontes de citocininas sobre o desenvolvimento do sistema assimilatório das plantas durante o cultivo in vitro. Apenas ZEA aumentou o número de feixes vasculares nas folhas dessa espécie. Segundo ALONI (2001), as citocininas atuam sobre a formação de células precursoras do sistema vascular e desempenham papel importante no aumento da sensibilidade dessas células à auxina, estimulando indiretamente o processo de diferenciação dos vasos.

O maior percentual de explantes com brotações foi verificado no tratamento com BAP e ZEA (dados não mostrados). ZEA, seguida de BAP e KIN, induziram a formação de maior número de brotações por explante, entretanto, somente a ZEA estimulou o desenvolvimento de multibrotações nessa espécie. BAP e KIN promoveram o maior crescimento em altura das brotações, enquanto que o TDZ promoveu efeito 

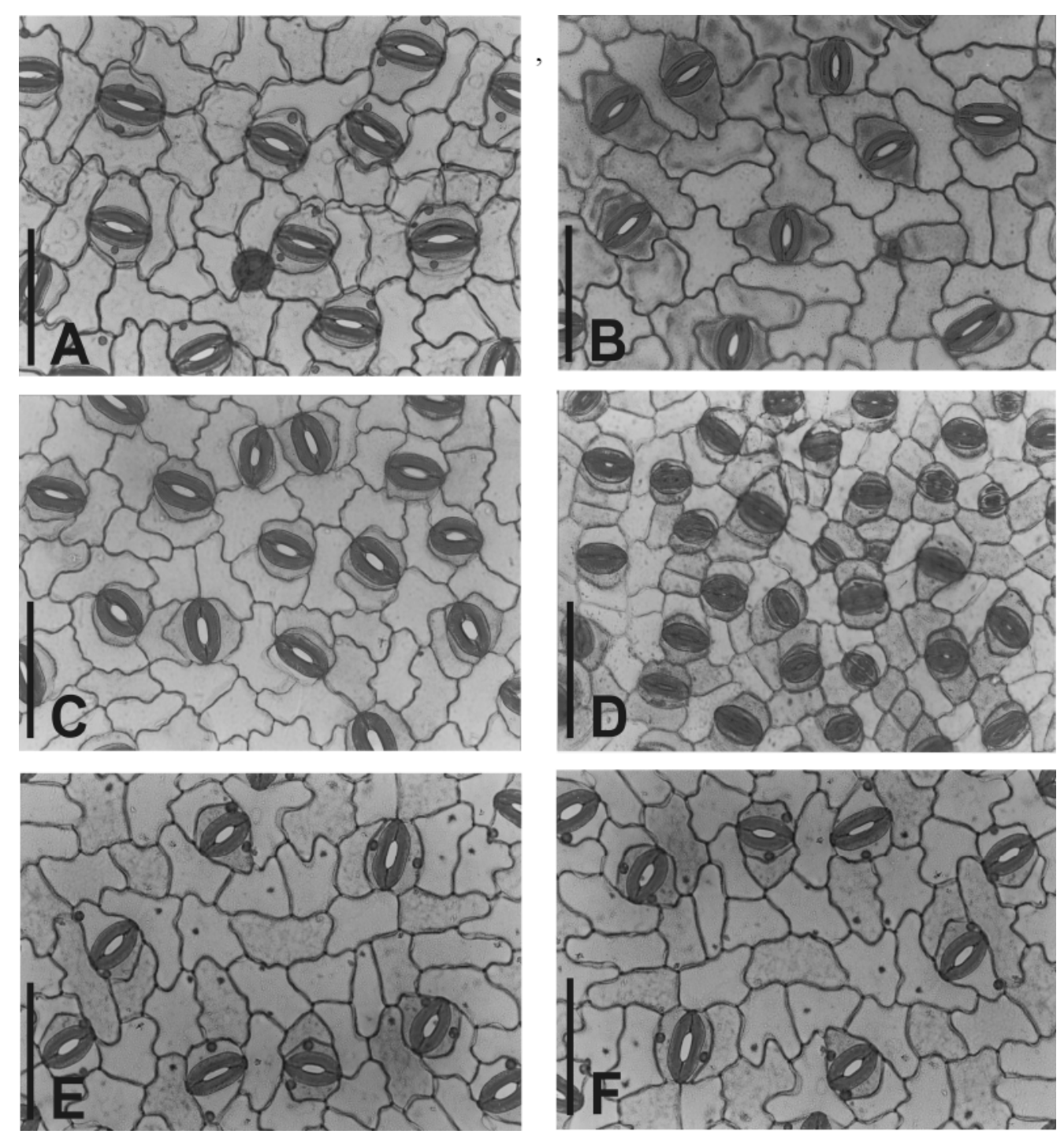

Figura 1 - Fotomicrografias de seções paradérmicas da superfície abaxial de folhas de A. glabra cultivada in vitro em meio WPM, acrescido de diferentes fontes de citocininas: (A) TDZ, (B) Controle (C) BAP, (D) ZEA, indicando elevada densidade estomática e presença de paredes celulares menos sinuosas (E e F) KIN. Barra $=50 \mu \mathrm{m}$.

inibitório sobre essa característica. A utilização de citocininas durante a fase de multiplicação reduziu indistintamente a emissão de raízes nas plantas, sobretudo naquelas cultivadas na presença de ZEA. Entretanto, BAP, KIN e ZEA proporcionaram o maior alongamento da maior raiz. KIN e BAP, além de promoverem maior crescimento das brotações durante a fase de multiplicação, induziram maior acúmulo de massa seca e maior desenvolvimento da área foliar durante a fase de aclimatização de A. glabra, possibilitando as maiores taxas de sobrevivência durante essa fase (dados não mostrados), o que pode ser reflexo do maior desenvolvimento dos parênquimas clorofilianos das plantas cultivadas na presença desses fitoreguladores.

\section{AGRADECIMENTOS}

Os autores agradecem à Fundação de Amparo à Pesquisa do Estado de Minas Gerais (FAPEMIG), pelo apoio financeiro para realização desse trabalho.

Ciência Rural, v.38, n.5, ago, 2008. 

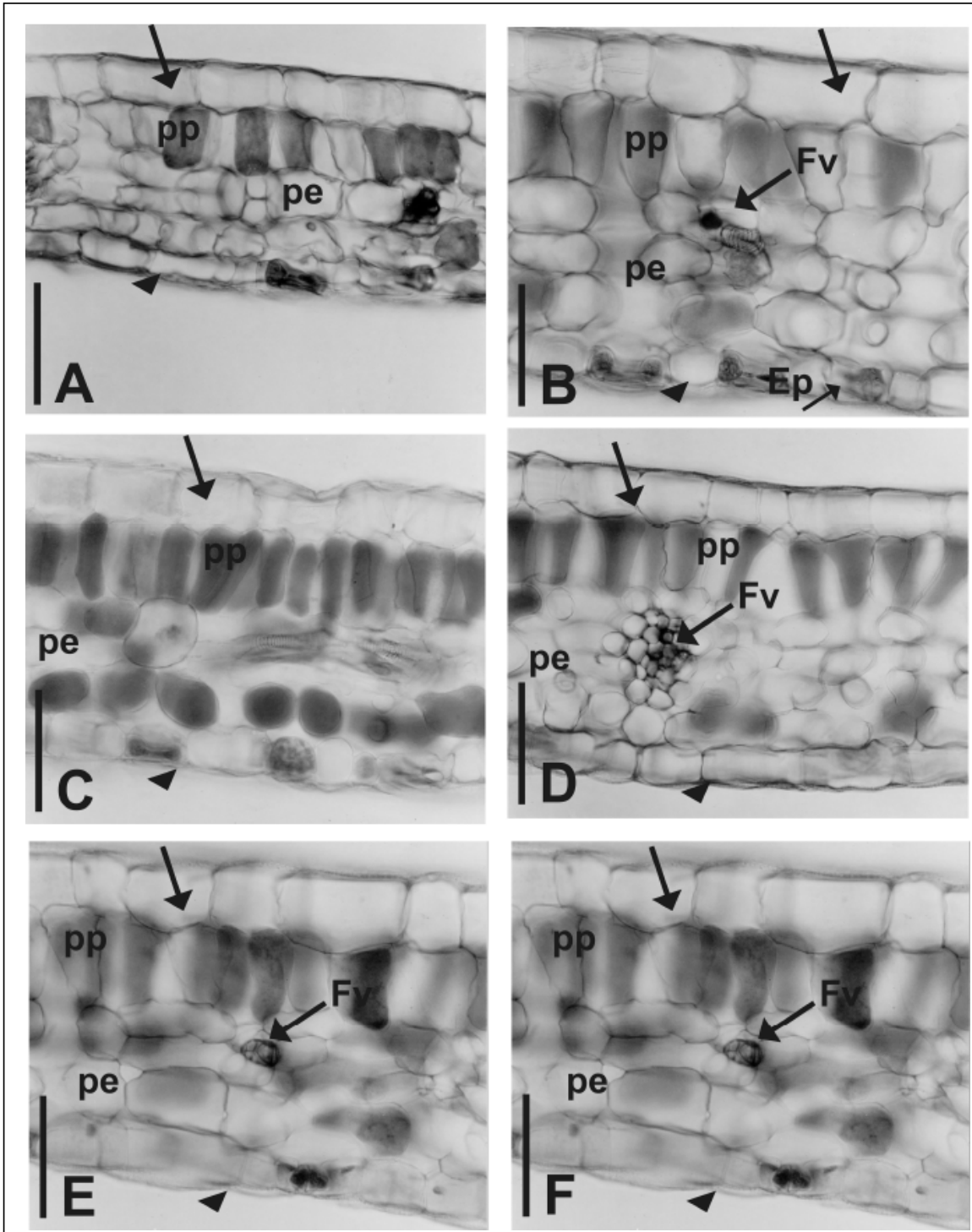

Figura 2 - Fotomicrografias de seções transversais de folhas de A. glabra cultivada in vitro, em meio WPM, acrescido de diferentes fontes de citocininas: (A) TDZ, (B) BAP, (C) KIN (D) ZEA, demonstrando a presença de feixe vascular com maior diâmetro e (E e F) Controle. Epiderme (Ep), parênquima paliçádico (pp), parênquima esponjoso (pe), feixe vascular (Fv), epiderme adaxial (setas) e epiderme abaxial (cabeças de seta). Barra $=50 \mu \mathrm{m}$.

\section{REFERÊNCIAS}

ALONI, R. Foliar and axial aspects of vascular differentiation: Hypotheses and evidence. Journal Plant Growth Regulation, New York, v.20, n.1, p.22-34, 2001.

CUTTER, E.G. Anatomia vegetal. 2.ed. São Paulo: Roca, 1986. 304p.

ENCINA, C.L. et al. In vitro morphogenesis of juvenile Annona cherimola Mill. Bud explants. Journal of Horticultural Science, Ashford, v.69, n.6, p.1053-1059, 1994.
JOHANSEN, D.A. Plant microtechnique. New York: McGraw-Hill, 1940. 523p.

KRAUS, J.E.; ARDUIN, M. Manual básico d métodos em morfologia vegetal. Rio de Janeiro: EDUR, 1997. 198p.

LEMOS, E.E.P.; BLAKE, J. Micropropagation of juvenile and mature Annona muricata L. Journal of Horticultural Science, Ashford, v.71, n.3, p.395-403, 1996.

LLOYD, G.; MCCOWN, B. Commercially-feasible micropropagation of Mountain laurel, Kalmia latifolia, by use 
of shoot tip culture. International Plant Propagation Society Proceedings, Washington, v.30, p.421-427, 1980.

NAGORI, R.; PUROHIT, S.D. In vitro plantled regeneration in Annona squamosa L. through direct shoot bud differentiation on hypocotyl segments. Scientia Horticulturae, Amsterdam, v.99, n.1, p.89-98, 2004.
SANTANA, J.R.F. Controle da Morfogênese in vitro em algumas espécies de Annonaceae. 2003. 237f. Tese (Doutorado em Fisiologia Vegetal) - Universidade Federal de Lavras, Lavras, MG

SCIUTTI, B.R.; MORINI, S. Water loss and photosynthesis of plum plantlets is influenced by relative humidity during rooting in vitro. Journal of Horticultural Science, Ashford, v.70, n.2, p.221-228, 1995 . 